\title{
Whole MILC: generalizing learned dynamics across tasks, datasets, and populations
}

\author{
Usman Mahmood ${ }^{1}$, Md Mahfuzur Rahman ${ }^{1}$, Alex Fedorov ${ }^{2}$, Noah Lewis ${ }^{2}$, \\ Zening $\mathrm{Fu}^{1}$, Vince D. Calhoun ${ }^{1,2,3}$, and Sergey M. Plis ${ }^{1}$ \\ 1 Georgia State University \\ \{umahmood1,mrahman21\}@student.gsu.edu, \{zfu,vcalhoun, splis\}@gsu.edu \\ ${ }^{2}$ Georgia Institute of Technology \\ af edorov@gatech.edu, 1hd231@gmail.com \\ 3 Emory University \\ Atlanta, GA, USA
}

\begin{abstract}
Behavioral changes are the earliest signs of a mental disorder, but arguably, the dynamics of brain function gets affected even earlier. Subsequently, spatio-temporal structure of disorder-specific dynamics is crucial for early diagnosis and understanding the disorder mechanism. A common way of learning discriminatory features relies on training a classifier and evaluating feature importance. Classical classifiers, based on handcrafted features are quite powerful, but suffer the curse of dimensionality when applied to large input dimensions of spatio-temporal data. Deep learning algorithms could handle the problem and a model introspection could highlight discriminatory spatio-temporal regions but need way more samples to train. In this paper we present a novel self supervised training schema which reinforces whole sequence mutual information local to context (whole MILC). We pre-train the whole MILC model on unlabeled and unrelated healthy control data. We test our model on three different disorders (i) Schizophrenia (ii) Autism and (iii) Alzheimers and four different studies. Our algorithm outperforms existing self-supervised pre-training methods and provides competitive classification results to classical machine learning algorithms. Importantly, whole MILC enables attribution of subject diagnosis to specific spatio-temporal regions in the fMRI signal.
\end{abstract}

Keywords: Transfer Learning · Self-Supervised · Deep Learning · Resting State fMRI.

\section{Introduction}

Mental disorders manifest in behavior that is driven by disruptions in brain dynamics 124]. Functional MRI captures the nuances of spatio-temporal dynamics that could potentially provide clues to the causes of mental disorders and enable early diagnosis. However, the obtained data for a single subject is of high dimensionality $m$ and to be useful for learning, and statistical analysis, one needs to collect datasets with a large number of subjects $n$. Yet, for any 
kind of a disorder, demographics or other types of conditions, a single study is rarely able to amass datasets large enough to go out of the $m \gg n$ mode. Traditionally small data problem is approached by handcrafting features [18] of much smaller dimension, effectively reducing $m$ via dimensionality reduction. Often, the dynamics of brain function in these representations vanishes into proxy features such as correlation matrices of functional network connectivity (FNC) [33.

Our goal is to enable the direct study of brain dynamics in the $m \gg n$ situation. In the case of brain data it, in turn, can enable an analysis of brain function via model introspection. In this paper, we show how one can achieve significant improvement in classification directly from dynamical data on small datasets by taking advantage of publicly available large but unrelated datasets. We demonstrate that it is possible to train a model in a self-supervised manner on dynamics of healthy control subjects from the Human Connectome Project (HCP) 32] and apply the pre-trained model to a completely different data collected across multiple sites from healthy controls and patients. We show that pre-training on dynamics allows the encoder to generalize across a number of datasets and a wide range of disorders: schizophrenia, autism, and Alzheimer's disease. Importantly, we show that learnt dynamics generalizes across different data distributions, as our model pre-trained on healthy adults shows improvements in children and elderly.

\section{Related Work}

Unsupervised pre-training is a well-known technique to get a head start for the deep neural network [9]. It finds wide use across a number of fields such as computer vision [13, natural language processing (NLP) 6] and automatic speech recognition (ASR) 22. However, outside NLP unsupervised pre-training is not as popular as supervised.

Recent advances in self-supervised methods with mutual information objectives are approaching performance of supervised training [26]16]2] and can scale pre-training to very deep convolutional networks (e.g., 50-layer ResNet). They were shown to benefit structural MRI analysis [10, learn useful representations from the frames in Atari games [1] and for speaker identification [28]. Pre-trained models can outperform supervised methods by a large margin in case of small data [13].

Earlier work in brain imaging 2027] have been based on unsupervised methods to learn the dynamics and structure of the brain using approaches such as ICA [3] and HMM [8]. Deep learning for capturing the brain dynamics has also been previously proposed $14[15[19$. In some very small datasets, transfer learning was proposed for use in neuroimaging applications 25|21/30. Yet another idea is the data generating approach [31]. ST-DIM [1] has been used for pre-training on unrelated data with subsequent use for classification [24]. 


\section{MILC}

We present MILC as an unsupervised pre-training method. We use MILC to pretrain on large unrelated and unlabelled data to better learn data representation. The learnt representations are then used for classification on downstream tasks adding a simple linear network on top of the pre-training architecture. The fundamental idea of MILC is to establish relationship between windows (a time slice from the entire sequence) and their respective sequences through learning useful signal dynamics. In all of our experiments we use encoded rsfMRI ICA time courses as our sequences and a consecutive chunk of time points as windows. The model uses the idea to distinguish among sequences (subjects) which proves to be extremely useful in downstream tasks e.g classification of $\mathrm{HC}$ or SZ subjects. To realize the concept, we maximize the mutual information of the latent space of a window and the corresponding sequence as a whole.

Let $D=\left\{\left(u_{t}^{i}, v^{j}\right): 1 \leq t \leq T, 1 \leq i, j \leq N\right\}$ be a dataset of pairs computed from ICA time courses. $u_{t}^{i}$ is the local embedding of $t$-th window taken from sequence $i, v^{j}$ is the global embedding for the entire sequence $j . T$ is the number of windows in a sequence, and $N$ is the total number of sequences. Then $D^{+}=$ $\left\{\left(u_{t}^{i}, v^{j}\right): 1 \leq t \leq T, i=j\right\}$ is called a dataset of positive pairs and $D^{-}=$ $\left\{\left(u_{t}^{i}, v^{j}\right): 1 \leq t \leq T, i \neq j\right\}$ - of negative pairs. The dataset $D^{+}$refers to a joint distribution and $D^{-}$- a marginal distribution of the whole sequence and the window in the latent space. Eventually, the lower bound with InfoNCE estimator [26] $\mathcal{I}_{f}\left(D^{+}\right)$is defined as:

$$
\mathcal{I}\left(D^{+}\right) \geq \mathcal{I}_{f}\left(D^{+}\right) \triangleq \sum_{i=1}^{N} \sum_{t=1}^{T} \log \frac{\exp f\left(\left(u_{t}^{i}, v^{i}\right)\right)}{\sum_{k=1}^{N} \exp f\left(\left(u_{t}^{i}, v^{k}\right)\right)}
$$

where $f$ is a critic function. Specifically, we are using separable critic $f\left(u_{t}, v_{s}\right)=$ $\phi\left(u_{t}^{i}\right)^{\top}\left(v^{j}\right)$, where $\phi$ is some embedding function parameterized by neural networks. Such embedding function is used to calculate value of a critic function in same dimensional space from two dimensional inputs. Critic learns an embedding function such that critic assigns higher values for positive pairs compared to negative pairs: $f\left(D^{+}\right) \gg f\left(D^{-}\right)$.

Our critic function takes the latent representation of a window and sequence as input. We define latent state of window as an output $z_{t}^{i}$ produced by the CNN part of MILC, given input from $t$-th window $x_{t}^{i}$ of sequence $i$. The latent state of sequence as $c^{j}$ is the global embedding obtained from MILC architecture. Thus the critic function for input pair $\left(x_{t}^{i}, x^{j}\right)$ - a window and a sequence - is $f=\phi\left(z_{t}^{i}\right)^{\top}\left(c^{j}\right)$. The loss is InfoNCE with $f$ as $L=-\mathcal{I}_{f}$. The scheme of the MILC is shown in Figure 1 .

\subsection{Transfer and Supervised Learning}

In the downstream task, we use the representation (output) of the attention model pre-trained using MILC as input to a simple binary classifier on top. Refer to section 4.1 for further details. 


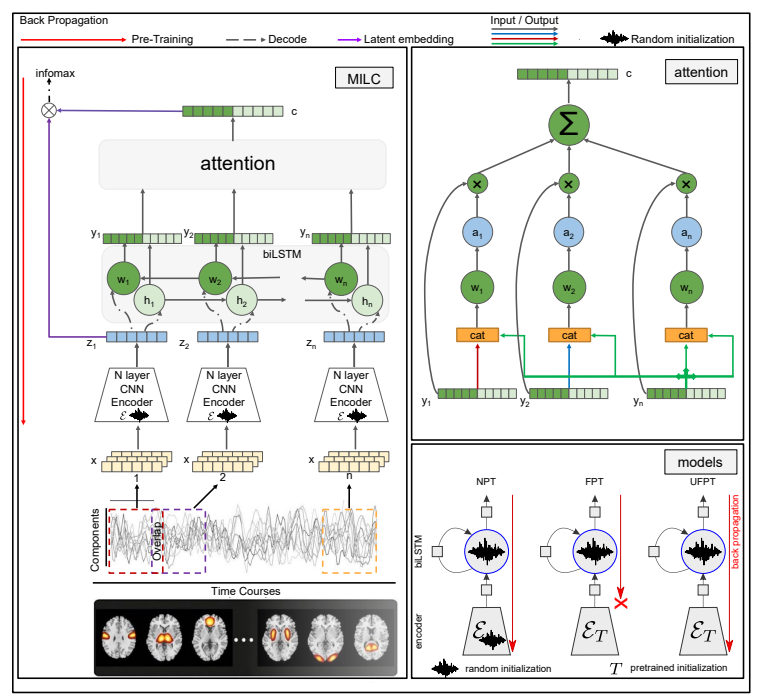

Fig. 1. Left: MILC architecture used in pre-training. ICA time courses are computed from the rsfMRI data. Results contain statistically independent spatial maps (top) and their corresponding time courses. Right Up: Detail of attention model used in MILC. Right Down: Three different models are used for downstream tasks.

\section{Experiments}

In this section we study the performance of our model on both, synthetic and real data. To compare and show the advantage of pre-training on large unrelated dataset we use three different kind of models - 1) FPT (Frozen Pre-Trained): The pre-trained model is not further trained on the dataset of downstream task, 2) UFPT (Unfrozen Pre-Trained): The pre-trained model is further trained on the dataset of downstream task and 3) NPT (Not Pre-trained): The model is not pre-trained at all and only trained on the dataset of downstream task. The models are shown in Figure 1. In each experiment, we compare all three models to demonstrate the effectiveness of unsupervised pre-training.

\subsection{Setup}

The CNN Encoder of MILC for simulation experiment consists of 4 1D convolutional layers with output features $(32,64,128,64)$, kernel sizes $(4,4,3,2)$ respectively, followed by ReLU after each layer followed by a linear layer with 256 units. For real data experiments, we use $31 \mathrm{D}$ convolutional layers with output features $(64,128,200)$, kernel sizes $(4,4,3)$ respectively, followed by ReLU after each layer followed by a linear layer with 256 units. We use stride 1 for all of the convolution layers. We also test against autoencoder based pre-training for simulation experiment, for which we use the same CNN encoder as for MILC 
in the reduction phase. For the decoder, we use the reverse architecture of the encoder that result in $10 \times 20$ windows at the output.

In MILC based pre-training, for all possible pairs in the batch, we take feature $z$ from the output layer of CNN encoder. The latent representation of the entire time series is then passed through biLSTM. The output of biLSTM is used as input to the attention model to get a single vector $c$, which represents the entire time series. Scores are calculated using $z$ and $c$ as explained in 3 . Using these scores, we compute the loss. The neural networks are trained using Adam optimizer.

In downstream tasks we are more interested in subjects for classification task, for each subject the output of attention model $(c)$ is used as input to a feed forward network of two linear layers with 200 and 2 units to perform binary classification. For experiments, a hold out is selected for testing and is never used through the training/validation phase. For each experiment, 10 trials are performed to ensure random selection of training subjects and, in each case, the performance is evaluated on the hold out (test data). The code is available at: https://github.com/UsmanMahmood27/MILC

\subsection{Simulation}

To generate synthetic data, we generate multiple 10-node graphs with $10 \times 10$ stable transition matrices. Using these we generate multivariate time series with autoregressive (VAR) and structural vector autoregressive (SVAR) models [23.

50 VAR times series with size $10 \times 20000$ are split into three time slices respectively for training, validation and testing. Using these samples, We pretrain MILC to assign windows to respective time series.

In the final downstream task, we classify the whole time-series into VAR or SVAR (obtained by randomly dropping 20\% VAR samples) groups. We generate 2000 samples and split as 1600 for training, 200 for validation and 200 for hold-out test. For both pre-training and downstream task, we follow the same set up as described in section 4.1 .

We compare the effectiveness of MILC with the model used in 24 and two variations of autoencoder based pre-training. The two variations of autoencoder are acquired by replacing the CNN encoder of 24] and MILC by the pre-trained or randomly initialized autoencoder during downstream classification, depending on the model as explained in section 4. We refer to these two variations as $A E_{-} S T D I M$ and $A E_{-} S T D I M+$ attention. Note that difference between the two is the added attention layer in the later during downstream classification.

It is observed that the MILC based pre-trained models can easily be fine-tuned only with small amount of downstream data. Note, with very few samples, models based on the pre-trained MILC $\overline{\text { FPT }}$ and UFPT) outperform the un-pre-trained models (NPT), ST-DIM models, autoencoder based models. ST-DIM based pre-training model 24] performs reasonably well compared to autoencoder and NPT models, however, MILC steadily outperforms ST-DIM. Results show that autoencoder based self-supervised pre-training does not assist in VAR vs. SVAR classification. Refer to Figure 2 Left for the results of simulation experiments. 


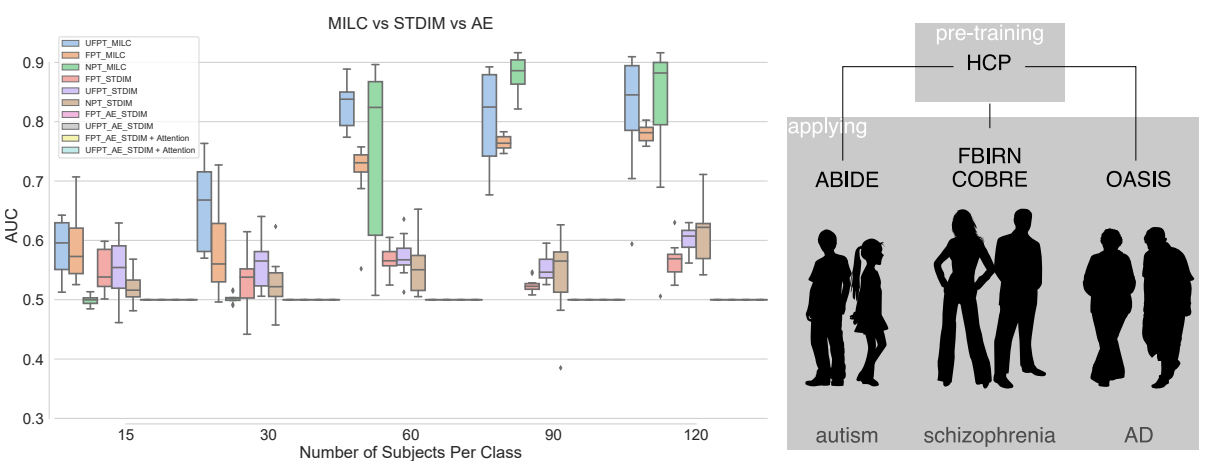

Fig. 2. Left: Area Under Curve (AUC) scores for VAR vs. SVAR time-series classification using MILC, ST-DIM and autoencoder based pre-training methods. MILC based pre-training greatly improves the performance of downstream task with small datasets. On the other side, ST-DIM works better than autoencoder based pre-training which completely fails to learn dynamics and thus exhibits poor performance. Right: Datasets used for pre-training and classification tasks. Healthy controls from the HCP [32] are used for pre-training guided by data dynamics alone ${ }^{1}$. The pre-trained model is then used in downstream classification tasks of 3 different diseases, 4 independently collected datasets, many of which contain data from a number of sites, and consist of populations with significant age difference. The age distributions in the datasets have the following mean and standard deviation: HCP: $29.31 \pm 3.67$; ABIDE: $17.04 \pm 7.29$; COBRE: $37.96 \pm 12.90 ;$ FBIRN: $37.87 \pm 11.25$; OASIS: $67.67 \pm 8.92$.

\subsection{Brain Imaging}

Datasets Next, we apply MILC to brain imagining data. We use rsfMRI data for all brain data experiments. Refer to Figure 2 for the details of the datasets used. We compare MILC with ST-DIM based pre-training shown in [24].

Four datasets used in this study are collected from FBIRN (Function Biomedical Informatics Research Network ${ }^{2}$ ) [17] project, from COBRE (Center of Biomedical Research Excellence) [5] project, from release 1.0 of ABIDE (Autism Brain Imaging Data Exchange ${ }^{3}$ [7] and from release 3.0 of OASIS (Open Access Series of Imaging Studies ${ }^{4}$ [29.

Preprocessing We preprocess the fMRI data using statistical parametric mapping (SPM12, http://www.fil.ion.ucl.ac.uk/spm/) under MATLAB 2016 environment. After the preprocessing, subjects were included in the analysis if the

\footnotetext{
${ }^{1}$ Human silhouettes are by Natasha Sinegina for Creazilla.com without modifications, https://creativecommons.org/licenses/by/4.0/

2 These data were downloaded from Function BIRN Data Repository, Project Accession Number 2007-BDR-6UHZ1.

${ }^{3}$ http://fcon_1000.projects.nitrc.org/indi/abide/

${ }^{4}$ https://www.oasis-brains.org/
} 
subjects have head motion $\leq 3^{\circ}$ and $\leq 3 \mathrm{~mm}$, and with functional data providing near full brain successful normalization [11].

For each dataset, 100 ICA components are acquired using the same procedure described in [11. However, only 53 non-noise components as determined per slice (time point) are used in all experiments. For all experiments, the fMRI sequence is divided into overlapping windows of 20 time points with $50 \%$ overlap along time dimension.

Schizophrenia For schizophrenia classification, we conduct experiments on two different datasets, FBIRN [17] and COBRE [5]. The datasets contain labeled Schizophrenia (SZ) and Healthy Control (HC) subjects.

FBIRN The dataset has total 311 subjects. We use two hold-out sets with sizes 32 and 64 for validation and test respectively, remaining are used for supervised training. The details of the results are shown in Figure 3 . We see, the pre-trained MILC models outperform NPT and also ST-DIM based pre-trained models.

COBRE The dataset has total 157 subjects - a collection of $68 \mathrm{HC}$ and 89 affected with SZ. We use two hold-out sets of size 32 each for validation and test respectively. The remaining data is used for supervised training. The results in Figure 3 strengthen the efficiency of MILC. That is, with only 15 training subjects, FPT and UFPT perform significantly better than NPT having $\simeq 0.20$ difference in their median AUC scores.

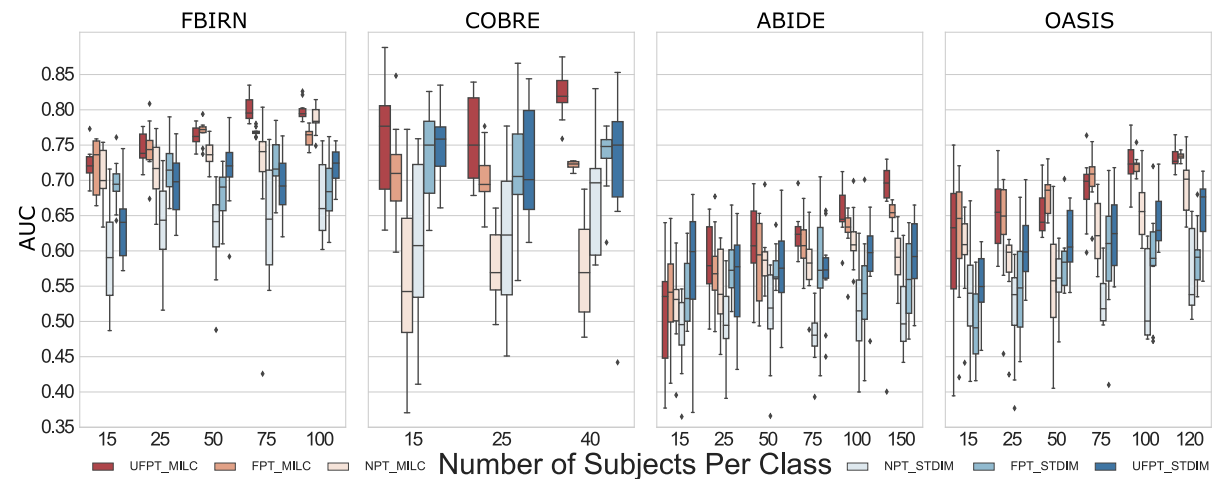

Fig. 3. AUC scores for all the three models (Refer to Figure 1) on real dataset. With every dataset, models pre-trained with MILC FPT UFPT perform noticeably better than not pre-trained model (NPT). Results also show that the learnability of MILC model dramatically increases with small increase in training data (x_axis). As we can see across the datasets, MILC outperforms ST-DIM with a large margin offering $\sim 10 \%$ higher AUC when maximum achievable AUC scores are compared. 
Autism With 569 total subjects, 255 are HC and 314 are affected with autism. We use 100 subjects each for validation and test purpose. The remaining data is used for downstream training i.e., autism vs. HC classification. Figure 3 shows, MILC pre-trained models perform reasonably better than NPT and thus reinforces our hypothesis that unsupervised pre-training learns signal dynamics useful for downstream tasks. We suspect that the reason why pre-trained models do not work well for 15 subjects is that the dataset is much different than HCP. The big age gap between subjects of HCP and ABIDE is a major difference and 15 subjects are not enough even for pre-trained models. Refer to Figure 2 for the demographic information of all the datasets.

Alzheimer's disease The dataset OASIS [29] has total 372 subjects with equal number (186) of HC and AZ patients. We use two hold-out sets each of size 64 respectively for validation and test purpose. The remaining are used for supervised training. Refer to Figure 3 for results. The AUC scores of pre-trained models is higher than NPT starting from 15 subjects, even with 120 subjects NPTdoes not perform equally well.

\subsection{Saliency}

Our experiments demonstrate that with the whole MILC pre-training we're able to achieve reasonable prediction performance from complete dynamics even on small data. Importantly, we're now able to investigate what in the dynamics was the most discriminative (see Figure 4).

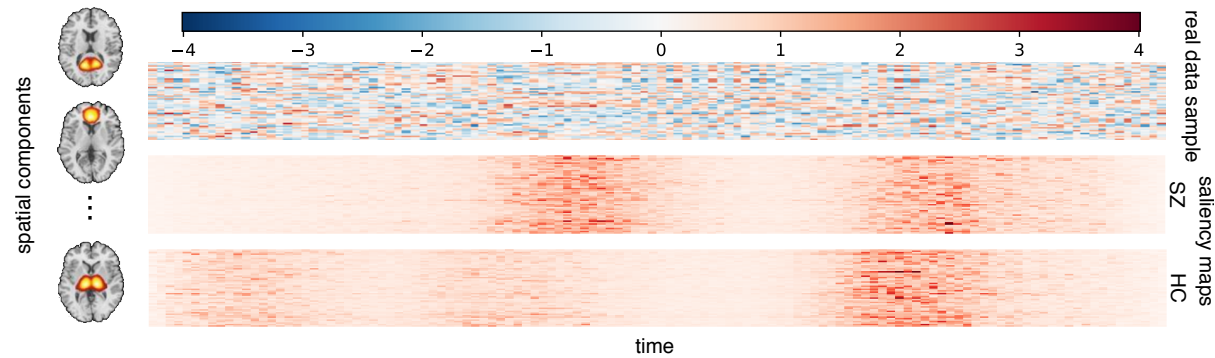

Fig. 4. Example saliency maps from a pre-trained MILC model: one for a healthy control and one for a schizophrenia subject (FBIRN data). More work is needed, but we already see that not only our model predicts diagnosis but also can point out when during the resting state scan discriminative activity was observed.

\section{Conclusions and Future Work}

As we have demonstrated, self-supervised pre-training of a spatio-temporal encoder gives significant improvement on the downstream tasks in brain imaging 
datasets. Learning dynamics of fMRI helps to improve classification results for all three dieseases and speed up the convergence of the algorithm on small datasets, that otherwise do not provide reliable generalizations. Although the utility of these results is highly promising by itself, we conjecture that direct application to spatio-temporal data will warrant benefits beyond improved classification accuracy in the future work. Working with ICA components is a smaller and thus easier to handle space that exhibits all dynamics of the signal, in future we will move beyond ICA pre-processing and work with fMRI data directly. We expect further model introspection to yield insight into the spatio-temporal biomarkers of schizophrenia. It may indeed be learning crucial information about dynamics that might contain important clues into the nature of mental disorders.

\section{Acknowledgement}

This study was in part supported by NIH grants 1R01AG063153 and 2R01EB006841. We'd like to thank and acknowledge the open access data platforms and data sources that were used for this work, including: Human Connectome Project (HCP), Open Access Series of Imaging Studies (OASIS), Autism Brain Imaging Data Exchange (ABIDE I), Function Biomedical Informatics Research Network (FBIRN) and Centers of Biomedical Research Excellence (COBRE).

\section{References}

1. Anand, A., Racah, E., Ozair, S., Bengio, Y., Côté, M.A., Hjelm, R.D.: Unsupervised state representation learning in Atari. arXiv preprint arXiv:1906.08226 (2019) 2

2. Bachman, P., Hjelm, R.D., Buchwalter, W.: Learning representations by maximizing mutual information across views. arXiv preprint arXiv:1906.00910 (2019) 2

3. Calhoun, V.D., Adali, T., Pearlson, G.D., Pekar, J.: A method for making group inferences from functional MRI data using independent component analysis. Human brain mapping 14(3), 140-151 (2001) 2

4. Calhoun, V.D., Miller, R., Pearlson, G., Adalı, T.: The chronnectome: time-varying connectivity networks as the next frontier in fmri data discovery. Neuron 84(2), 262-274 (2014) 1

5. Çetin, M.S., Christensen, F., Abbott, C.C., Stephen, J.M., Mayer, A.R., Cañive, J.M., Bustillo, J.R., Pearlson, G.D., Calhoun, V.D.: Thalamus and posterior temporal lobe show greater inter-network connectivity at rest and across sensory paradigms in schizophrenia. Neuroimage 97, 117-126 (2014) 6, 7

6. Devlin, J., Chang, M.W., Lee, K., Toutanova, K.: Bert: Pre-training of deep bidirectional transformers for language understanding. arXiv preprint arXiv:1810.04805 (2018) 2

7. Di Martino, A., Yan, C.G., Li, Q., Denio, E., Castellanos, F.X., Alaerts, K., Anderson, J.S., Assaf, M., Bookheimer, S.Y., Dapretto, M., et al.: The autism brain imaging data exchange: towards a large-scale evaluation of the intrinsic brain architecture in autism. Molecular psychiatry 19(6), 659 (2014) 6

8. Eavani, H., Satterthwaite, T.D., Gur, R.E., Gur, R.C., Davatzikos, C.: Unsupervised learning of functional network dynamics in resting state fmri. In: International Conference on Information Processing in Medical Imaging. pp. 426-437. Springer (2013) 2 
9. Erhan, D., Bengio, Y., Courville, A., Manzagol, P.A., Vincent, P., Bengio, S.: Why does unsupervised pre-training help deep learning? Journal of Machine Learning Research 11(Feb), 625-660 (2010) 2

10. Fedorov, A., Hjelm, R.D., Abrol, A., Fu, Z., Du, Y., Plis, S., Calhoun, V.D.: Prediction of progression to Alzheimers disease with deep InfoMax. arXiv preprint arXiv:1904.10931 (2019) 2

11. Fu, Z., Caprihan, A., Chen, J., Du, Y., Adair, J.C., Sui, J., Rosenberg, G.A., Calhoun, V.D.: Altered static and dynamic functional network connectivity in alzheimer's disease and subcortical ischemic vascular disease: shared and specific brain connectivity abnormalities. Human Brain Mapping (2019) 7

12. Goldberg, D.P., Huxley, P.: Common mental disorders: a bio-social model. Tavistock/Routledge (1992) 1

13. Hénaff, O.J., Razavi, A., Doersch, C., Eslami, S., Oord, A.v.d.: Data-efficient image recognition with contrastive predictive coding. arXiv preprint arXiv:1905.09272 (2019) 2

14. Hjelm, R.D., Calhoun, V.D., Salakhutdinov, R., Allen, E.A., Adali, T., Plis, S.M.: Restricted Boltzmann machines for neuroimaging: an application in identifying intrinsic networks. NeuroImage 96, 245-260 (2014) 2

15. Hjelm, R.D., Damaraju, E., Cho, K., Laufs, H., Plis, S.M., Calhoun, V.D.: Spatiotemporal dynamics of intrinsic networks in functional magnetic imaging data using recurrent neural networks. Frontiers in neuroscience 12, 600 (2018) 2

16. Hjelm, R.D., Fedorov, A., Lavoie-Marchildon, S., Grewal, K., Bachman, P., Trischler, A., Bengio, Y.: Learning deep representations by mutual information estimation and maximization. arXiv preprint arXiv:1808.06670 (2018) 2

17. Keator, D.B., van Erp, T.G., Turner, J.A., Glover, G.H., Mueller, B.A., Liu, T.T., Voyvodic, J.T., Rasmussen, J., Calhoun, V.D., Lee, H.J., et al.: The function biomedical informatics research network data repository. Neuroimage 124, 1074 1079 (2016) 6. 7

18. Khazaee, A., Ebrahimzadeh, A., Babajani-Feremi, A.: Application of advanced machine learning methods on resting-state fMRI network for identification of mild cognitive impairment and Alzheimer's disease. Brain Imaging and Behavior 10(3), 799-817 (Sep 2016). https://doi.org/10.1007/s11682-015-9448-7, https://doi.org/ 10.1007/s11682-015-9448-7/2

19. Khosla, M., Jamison, K., Kuceyeski, A., Sabuncu, M.R.: Detecting abnormalities in resting-state dynamics: An unsupervised learning approach. In: International Workshop on Machine Learning in Medical Imaging. pp. 301-309. Springer (2019) 2

20. Khosla, M., Jamison, K., Ngo, G.H., Kuceyeski, A., Sabuncu, M.R.: Machine learning in resting-state fMRI analysis. Magnetic resonance imaging (2019) 2

21. Li, H., Parikh, N.A., He, L.: A novel transfer learning approach to enhance deep neural network classification of brain functional connectomes. Frontiers in Neuroscience 12, 491 (2018). https://doi.org/10.3389/fnins.2018.00491, https: //www.frontiersin.org/article/10.3389/fnins.2018.00491|2

22. Lugosch, L., Ravanelli, M., Ignoto, P., Tomar, V.S., Bengio, Y.: Speech model pre-training for end-to-end spoken language understanding. arXiv preprint arXiv:1904.03670 (2019) 2

23. Lütkepohl, H.: New introduction to multiple time series analysis. Springer Science \& Business Media (2005) 5

24. Mahmood, U., Rahman, M.M., Fedorov, A., Fu, Z., Plis, S.: Transfer learning of fmri dynamics. arXiv preprint arXiv:1911.06813 (2019) 2, 5, 6 
25. Mensch, A., Mairal, J., Bzdok, D., Thirion, B., Varoquaux, G.: Learning neural representations of human cognition across many fMRI studies. In: Advances in Neural Information Processing Systems. pp. 5883-5893 (2017) 2

26. Oord, A.v.d., Li, Y., Vinyals, O.: Representation learning with contrastive predictive coding. arXiv preprint arXiv:1807.03748 (2018) 2,3

27. Plis, S.M., Hjelm, D., Salakhutdinov, R., Allen, E.A., Bockholt, H.J., Long, J.D., Johnson, H.J., Paulsen, J., Turner, J.A., Calhoun, V.D.: Deep learning for neuroimaging: a validation study. Frontiers in Neuroscience 8(229) (2014) 2

28. Ravanelli, M., Bengio, Y.: Learning speaker representations with mutual information. arXiv preprint arXiv:1812.00271 (2018) 2

29. Rubin, E.H., Storandt, M., Miller, J.P., Kinscherf, D.A., Grant, E.A., Morris, J.C., Berg, L.: A prospective study of cognitive function and onset of dementia in cognitively healthy elders. Archives of neurology 55(3), 395-401 (1998) 6, 8

30. Thomas, A.W., Müller, K.R., Samek, W.: Deep transfer learning for whole-brain fMRI analyses. arXiv preprint arXiv:1907.01953 (2019) 2

31. Ulloa, A., Plis, S., Calhoun, V.: Improving classification rate of schizophrenia using a multimodal multi-layer perceptron model with structural and functional MR. arXiv preprint arXiv:1804.04591 (2018) 2

32. Van Essen, D.C., Smith, S.M., Barch, D.M., Behrens, T.E., Yacoub, E., Ugurbil, K., Consortium, W.M.H., et al.: The WU-Minn human connectome project: an overview. Neuroimage 80, 62-79 (2013) 2, 6

33. Yan, W., Plis, S., Calhoun, V.D., Liu, S., Jiang, R., Jiang, T.Z., Sui, J.: Discriminating schizophrenia from normal controls using resting state functional network connectivity: A deep neural network and layer-wise relevance propagation method. In: 2017 IEEE 27th International Workshop on Machine Learning for Signal Processing (MLSP). pp. 1-6. IEEE (2017) 2 